\title{
Adopting a communication lifestyle
}

\author{
N. S. Sharp $\odot^{*}$ \\ Sharp Science Communication Consulting, LLC, Denver, Colorado 80210, USA
}

(Received 16 June 2020; accepted 1 October 2020; published 24 November 2020)

\begin{abstract}
As fluid dynamicists, good communication is key to our success in academia, industry, and research. Yet our training in this critical skill is often lacking. In this article, I present a framework for the science communication process and techniques for integrating communication training into everyday practices. With some forethought and habit-building, preparing engaging conference presentations, writing journal articles, or interacting with journalists does not have to be a painful, last-minute scramble. Instead, these activities can be just another part of your communication lifestyle.
\end{abstract}

DOI: 10.1103/PhysRevFluids.5.110515

\section{INTRODUCTION}

Every scientist and engineer is, by necessity, a communicator. In academia, research often occurs at interdisciplinary boundaries and requires collaboration between experts from vastly different technical backgrounds. Within fluid dynamics, it is not unusual to find overlaps between biology, medicine, robotics, and even paleontology. In industry, engineers frequently work in teams on largescale projects that require clear communication between members with different specializations.

Yet communication skills often receive short shrift in our education. A 2010 survey by the American Society of Mechanical Engineers found that industry managers consider entry-level engineers lacking in both oral and written communication skills [1]. Despite increased attention to technical communication in engineering education, discrepancies remain between the skills expected by industry and those that are taught and practiced in the academic curriculum [2,3].

Simultaneously, social media has exploded with users interested in science communication, and many scientists now find themselves using these platforms to discuss, promote, and engage with others about scientific content.

The term "science communication" itself is broad and not well defined. As a field of academic research, social scientists disagree as to whether science communication constitutes its own independent discipline [4,5]. In practice, science communication is sometimes conflated with science outreach and viewed as a vehicle for scientists explaining science to a public audience. But although communicating with the public is an aspect of science communication, those activities do not represent the sum of science communication. As Burns et al. [4] identify in their own definition, "Science communication may involve science practitioners, mediators, and other members of the general public, either peer-to-peer or between groups." In other words, even standard academic activities-like journal publication and conference presentations, as well as the team-based activities of industry-constitute science communication.

\footnotetext{
"nicole.sharp@gmail.com
}

Published by the American Physical Society under the terms of the Creative Commons Attribution 4.0 International license. Further distribution of this work must maintain attribution to the author(s) and the published article's title, journal citation, and DOI. 

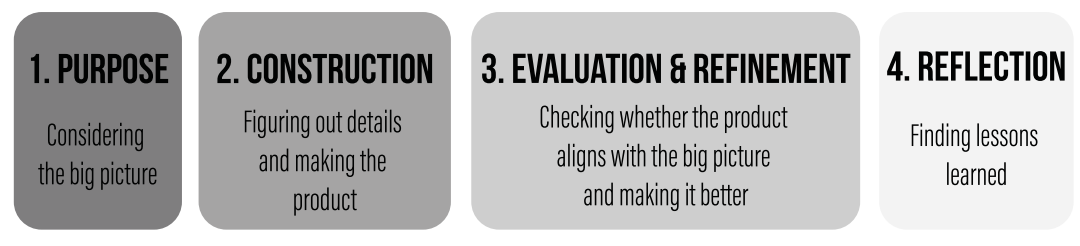

FIG. 1. The four phases of the science communication process.

In my own experience, communicating science-both to the public and my academic colleagues - has been a rewarding way to strengthen my oral and written communication skills and build subject expertise. The skills that underlie good science communication include compelling, understandable visuals; clear, concise, and technically accurate explanations; and the use of narrative structures. Regardless of one's final audience, the best technical communicators exploit these skills; to become better communicators, we must develop such skills through deliberate practice [6].

Due to the wide range of fields touched by fluid dynamics and the increasingly interdisciplinary nature of research in the subject, fluid dynamicists require strong communication skills to succeed, regardless of their interest in outreach or public engagement. This article aims to improve communication skills within the fluid dynamics research community by providing techniques and resources for integrating communication training and practice into regular research activities. Rather than dedicating attention to writing and speaking about science only to the weeks before manuscript submission or conference deadlines, we should instead build habits that acknowledge the integral nature of communication to our work.

To that end, in Sec. II, I introduce an overview of the science communication process and the questions each communicator should answer when embarking on a project. Habit-building forms the basis for Sec. III, which is divided into subsections around individual and group-based practices. The former focuses on general habit-building around planning and critiquing work, and the latter suggests specific methods for integrating science communication training into regular group meetings. I also illustrate principles of good communication with several examples from the fluid dynamics community. Together these efforts can support a better communication lifestyle for both students and professionals.

\section{SCIENCE COMMUNICATION PROCESS}

In my projects, I break down the process of developing any communication product into four phases, as illustrated in Fig. 1 [7]. The first phase, purpose, focuses on establishing guiding principles for the rest of the process by answering three key questions:

(1) What is my goal?

(2) Who is my audience?

(3) What is my message?

Every communication effort has an underlying motivation, whether one is a researcher making a Gallery of Fluid Motion video or an engineer writing a white paper to secure funding. Developing a final product that supports that goal requires explicitly identifying what communicators want to accomplish. If their goal, for example, is to recruit new graduate students, then aiming their department seminar to the senior-most scientists in attendance is unlikely to help.

The audience is another critical component to consider. As undergraduates, students write for an audience of one-their professor-with the singular goal of demonstrating their knowledge. For graduate students, researchers, and industry members, this is no longer the case. Even when addressing a technical audience, the savvy communicator must consider the range of experience and familiarity their audience has with the topic, as well as what the appropriate tone, level of detail, and use of terminology should be. 
Finally, communicators must consider their message. They may have several key points to make, but whether they are producing a blog post or a textbook, there should always be one overarching message a reader or listener should leave understanding. That message serves as the keystone about which the work is structured. In beginning composition, writers typically think of this message as their thesis statement. In the world of research, the key message often concerns the implications of a research project's results.

I use this exercise of answering three purpose questions regularly, whether I am developing a presentation, writing an article, or preparing a YouTube video. It is also an exercise I ask each of my clients to complete as we determine the form and scope of any project. Those familiar with science communication and scientific writing guides will recognize that these three questions echo many other authors' recommendations [8-10]. For those accustomed to simply opening a document or presentation and typing, taking the time to complete this exercise can save future heartache and revision later.

The second phase of the science communication process is construction, where the hard work of making version 1.0 takes place. The details of this phase depend on the type of product the communicator is making, so I will not elaborate here. Where the purpose phase is about determining the bigger picture, this phase takes place "down in the weeds," dealing with the practical details of writing a paper, preparing a poster, or producing a video.

Once a first version of the product is complete, it is time for the third phase, evaluation and refinement. In this stage, the communicator returns to the bigger picture determined in the purpose phase and asks if their creation will help achieve their goal and whether their audience will understand the work and its message as intended. To evaluate that aspect of a work-in-progress, I highly recommend test audiences, friendly review [10], and/or peer review from those unfamiliar with the work. I will return to those topics in Sec. III, and here merely note that this outside perspective is critical to revising and refining any product before it is ready for release.

The final stage of the science communication process, reflection, typically takes place after a project is completed, e.g., the paper published, presentation given, or video uploaded. In this stage, I encourage communicators to take time to appreciate what worked and what did not, lessons learned for the future, and, importantly, what skills they have gained or improved as they completed the project.

Taken altogether, the science communication process I present here is extremely general and may be adapted to a variety of projects. It is, by no means, the sole way to approach science communication, but it is a construct that has helped me, my clients, and my students. With this framework in mind, I now turn to some specifics of communication habit-building.

\section{COMMUNICATION HABIT-BUILDING}

Communicating science is a complex cognitive skill that integrates planning, knowledge synthesis and transformation, language production, and idea review, often simultaneously. From a psychological perspective, meeting these substantial demands requires deliberate practice as Kel$\operatorname{logg}$ and Whiteford [6] describe for training advanced writing skills (emphasis in original):

"Becoming an expert writer entails gaining control over perceptual, motor, and cognitive processes so that one can respond adaptively to the specific needs of the task at hand, just as a professional violinist or basketball player must do. This occurs by reducing the demands that relevant processes make on the limited resources of executive attention and working memory storage. For the skill as a whole to be well controlled, its component processes must become relatively automatic and effortless through practice. The term deliberate practice refers to practice undertaken with a specific goal to improve."

Thus, when choosing communication exercises or habits to pursue, consider how the activity will help the growing communicator reduce or manage the myriad cognitive loads they are juggling. Some communicators - both native and non-native English speakers-will benefit from rote, sentence-level revision exercises (Sec. III B 6), whereas others may find planning exercises most 
useful (Sec. III A 1). To put matters more colloquially, with any exercise suggested here, your mileage may vary. I encourage readers, regardless of their communication experience level, to sample widely to discover what works.

In the next two subsections, I recommend habits and exercises to pursue both individually and as a group.

\section{A. Habit-building as an individual}

One hurdle for many inexperienced communicators is recognizing the extensive process behind a finished product. As Heard [10] describes, "Most writers struggle. I didn't realize this because I had been seeing their writing product, not their writing process, which led to finished work that was clear, smooth, and easy to understand" (emphasis in original). This need to share process and not simply the product is one reason I have developed the science communication process outlined in Sec. II. It is also why I encourage professors to let their students see their own working process, not just a finalized presentation or grant proposal.

The following recommendations and exercises are aimed at individuals. Although anyone may benefit from them, the first two sections are most beneficial for students, whereas the final section is aimed more at those in advisory roles.

\section{Planning}

In my experience, planning is the most crucial (and often absent) piece of the process for students. For this reason, I emphasize the purpose phase described in Sec. II in my science communication workshops. Every project I undertake-including preparing this paper and the talk that preceded it—begins by setting aside time to consider my goals, audience, and message well before I pick up a pen or sit at the keyboard to start an outline. Others may find that wordstacks or concept maps provide a more useful starting point for their planning; see Chapter 7 of [10] for descriptions of those and other planning techniques.

To avoid procrastination or becoming stuck in the initial planning phase, it is helpful to break large projects down by setting intermediate deadlines. For example, submitting a Gallery of Fluid Motion video involves two external deadlines: the abstract submission and the final video submission. But to ensure they meet those deadlines with a good product, a researcher could set themselves a series of internal deadlines including: answering the three purpose questions; writing a script; finalizing a storyboard; completing filming; creating a rough cut; receiving feedback from test audiences; and finalizing the video. Intermediate deadlines not only help produce a better product, they counter the sense of being overwhelmed by turning a large task into a series of smaller, more manageable ones.

\section{Getting started}

For those who still struggle to get words onto the page-due to strong self-editing, for examplefreewriting exercises can help. The ground rules are simple: after determining what information belongs in the next section, set a timer-five minutes is a good starting point-and start writing. Stopping and rereading what is written are not allowed. Revision is not allowed until later. Often a few minutes of this exercise is enough to give writers the momentum needed to continue. Not everything produced this way will be worth keeping, but freewriting can help writers get past a dreaded first draft and into the potentially easier task of revision.

\section{Revision and critique}

For others - chiefly, instructors or evaluators-revision is dreaded due to the time requirements of providing critique. But there are several avenues to help senior researchers to avoid becoming overwhelmed by giving feedback. One is peer review, which in writing circles is different than the peer review to which scientists are accustomed; I will return to peer review in Sec. III B 8. 
Changing one's critiquing techniques can also reduce the time needed to give feedback. Often professors attempt to provide detailed written feedback on large sections of a student's work. In some cases, the student receives a draft dripping with red ink; in others, professors choose to simply rewrite the draft entirely. Both methods involve a large time commitment for the evaluator and provide the student with little room to grow as a communicator.

To see why, consider the technical equivalent: a student comes to office hours because they are struggling with a problem set. Rather than sitting down with the student and identifying specific issues, the instructor simply hands them the solution key and sends them away. Most of us would recognize this as a poor recipe for learning fluid dynamics, yet this is exactly how communication feedback is often treated.

Instead, I suggest an alternative method for critique, based on techniques I was taught as a peer writing tutor. Rather than attempting to fix every mistake at once, examine a draft for major flaws or repeated mistakes. Perhaps the writer's verbosity makes their logic vague and hard to follow. Find a passage that exemplifies these issues and discuss that passage together. Point out the problem and guide the student into finding their own clearer rephrasing, perhaps prompted by a few suggestions. After repeating this process of identifying problems and finding solutions for a subsection of the draft, encourage the student to continue the process on their own for the remainder of the draft before returning for additional help.

This methodology is helpful in two ways: it saves the evaluator time they would have spent on extensive correction, and it places responsibility for the work back into the student's hands, allowing them to learn both how to identify and to correct issues on their own. Without that opportunity, the student will likely continue making the same mistakes in subsequent projects.

In particular, students who are non-native speakers of English may struggle when writing and presenting in technical English. Remember that university writing centers are an excellent resource; if evaluators can help a student identify a specific, persistent problem, the student can then look for help from the writing center to address it.

Working one-on-one or individually is one way to build communication habits, but the rewards are even richer when working as a group.

\section{B. Habit-building as a group}

Regular research group meetings are an excellent venue for practicing communication skills together. Simply integrating a communication exercise into each meeting provides all group members regular training and deliberate practice to advance their mastery. Even outside traditional group meetings, informal student groups can effectively improve communication skills [11].

The following exercises and resources are not an exhaustive list, but they should provide a good starting point. I present them, roughly, in an order corresponding to the science communication process presented in Sec. II, beginning with those useful for the purpose phase.

\section{Audience adaptation}

Inexperienced communicators can struggle with identifying their audience and recognizing how audience affects the level of detail, jargon, and tone they should use. Figure 2 shows how Dickerson et al. [12] explain their study of mammals shedding water through shaking to three different audiences. For an academic audience, the authors use more specialized language and longer, more complex sentence structures. In a message aimed toward politicians, they simplify their sentences and concentrate on useful applications of their work while still including the most significant results. In a book for the general public, Hu uses even less formal language and frames the work in the context of a story about one specific dog.

In a group format, it is useful to discuss these issues explicitly-highlighting, for example, the differences between speaking to other laboratory members, a conference audience, a classroom of high schoolers, or a politician. Then the group can take a single message, like a description of the laboratory's focus or a particular member's latest work, and subdivide into smaller groups, each 


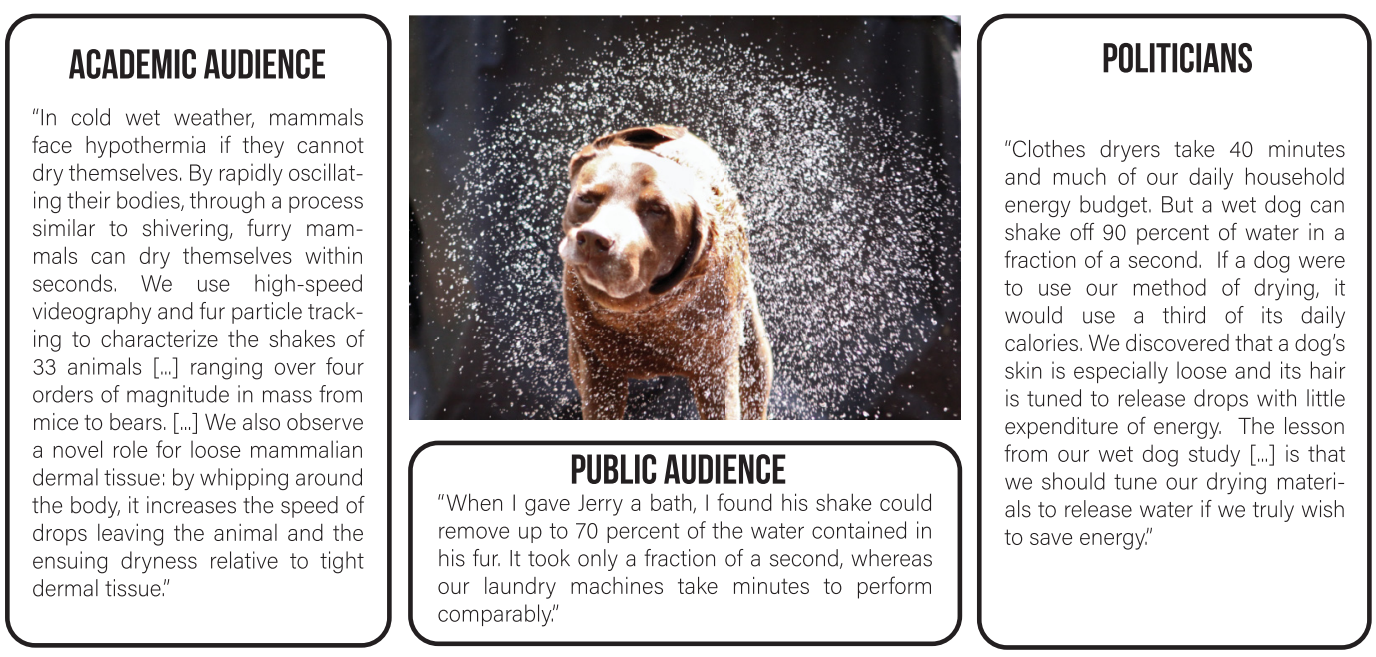

FIG. 2. Explanations of mammals shaking themselves dry aimed at three different audiences: academic audiences [12], the public [13], and politicians [14]. Wet dog image courtesy of A. Dickerson and D. Hu, used with permission.

tasked with adapting that message for a different, specific audience. Afterwards, each subgroup can present their results and the group as a whole can discuss just how the message changes. Exercises like this allow all members to participate, even in a fairly large group, and feature the interactivity and discussion communication researchers Silva and Bultitude [15] found are effective best practices in science communication training.

\section{Message discovery and refinement}

Discovering and refining a key message can be difficult, even for experienced communicators. Being familiar with every detail of a work makes it hard to step back and identify a single overarching message. For this, I recommend the "Half-Life Your Message" exercise [16]. The concept is simple: A speaker first explains their topic in 60 seconds, then immediately explains the same information in 30 seconds, in 15 seconds, and finally in 8 seconds. The exercise forces speakers to quickly refine their ideas down to the most critical message. It takes roughly three minutes total for any given speaker; a video timer for the exercise is available at [17].

In large groups, I first demonstrate the exercise myself using a topic suggested by participants. Then, we repeat the exercise several times in subgroups of three or four to allow each person a chance to speak, reflect, and give and receive feedback. Afterwards, the group as a whole discusses the results in terms of what worked and what did not, as well as whether they liked a particular version of their message. The entire exercise can be completed, even in a large classroom, in about 15 minutes. I have run this particular exercise with undergraduate students, graduate students, professors, and military officers at institutions across the United States, and it is consistently popular.

\section{Narrative structure}

In scientific writing and presenting, we use a standard narrative structure: introduction, methods, results, and discussion, known collectively as IMRaD [10]. Review papers, book chapters, and other scientific writing can deviate from this structure, but it is by far the most familiar. However, outside of journal articles, it is often not the most useful or engaging structure for communicating science.

Especially when taking scientific work to more general audiences-including to a grant proposal committee or a journalist-it is worth considering alternative narrative structures that, unlike 


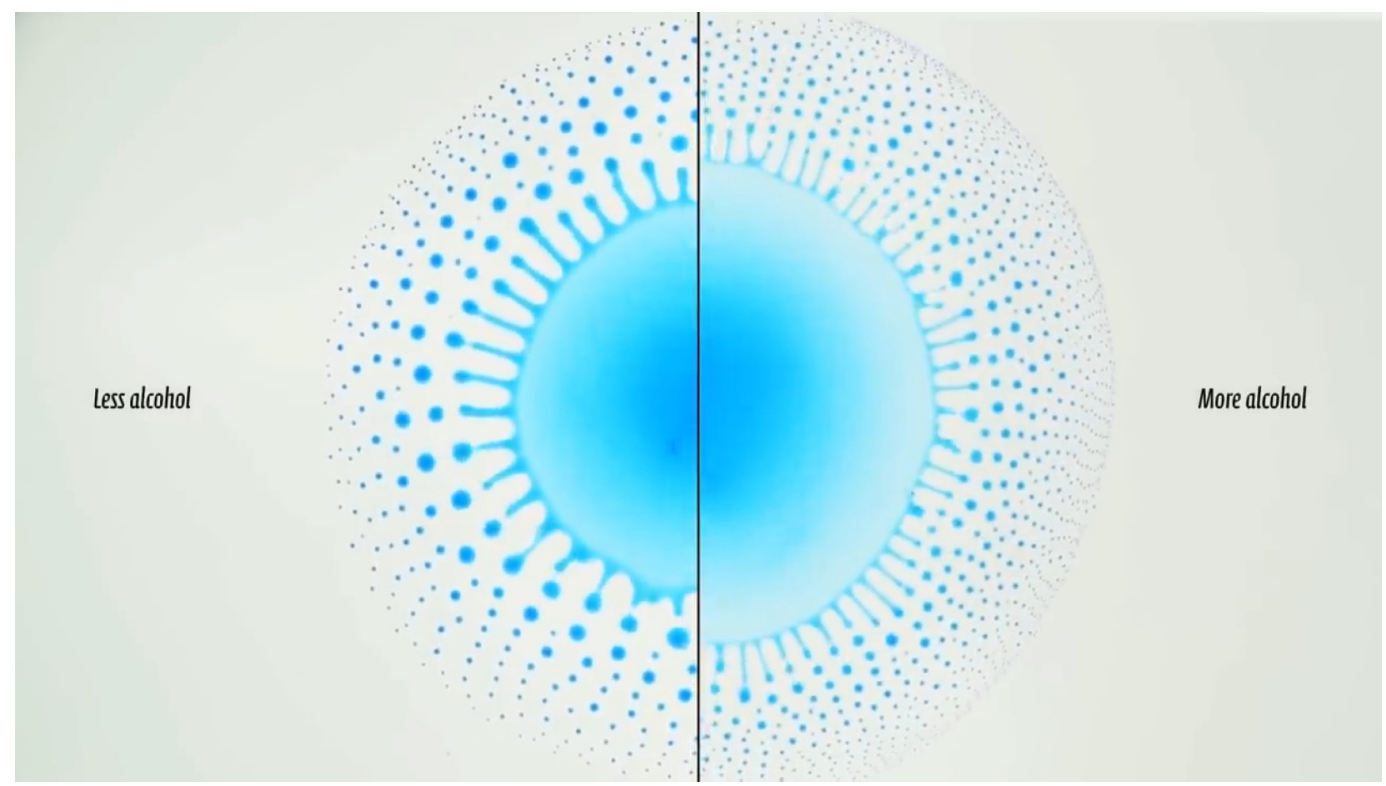

FIG. 3. Comparing two results as complementary halves of a figure allows viewers to quickly and easily identify differences. Screenshot from Ref. [22], used with permission.

IMRaD, return the focus to the characters engaged in the narrative, namely, the scientists. The Hero's Journey - the narrative structure behind stories like The Lord of the Rings, Harry Potter, and Star Wars: A New Hope-is particularly adaptable to telling scientific stories [18]. My recorded 2018 talk on this subject is available at [19].

For those interested in a deeper dive into narrative structure, science writers and journalists share the behind-the-scenes development of their articles on The Open Notebook website [20]. Hart [21] also provides extensive guidance on writing and structuring narrative nonfiction. To see these principles in action in the context of fluid dynamics, I highly recommend Ref. [13].

\section{Improving figures and visuals}

As a subject, fluid dynamics enjoys the advantage of striking and beautiful imagery suitable for both outreach and capturing media attention. But with this beauty comes challenges: researchers must find a balance between aesthetics and scientific rigor, and they must also contend with visual complexity that can easily confuse audiences.

The critical question to ask for any figure is what do I want my audience to see? A good scientific visual should be quickly and easily understandable, preferably without the need to repeatedly look between the figure and its caption. The context in which the figure is presented also matters. Consider a comparison between two results, a common visual in fluid dynamics. The standard approach to such a figure places one or more full images next to one another. This placement requires the viewer to glance back and forth between the images to recognize their differences and works well enough in a journal article where the reader can take the time they need to do so. But in a presentation or a Gallery of Fluid Motion video, the pacing is dictated for the viewer. To make a comparison clear more quickly, it helps to use complementary halves of an image like the ones in Fig. 3, from Durey et al.'s 2017 Milton Van Dyke award-winning video on bursting droplets driven by Marangoni effects [22]. By placing half of each example directly next to one another, even subtle differences between the two cases are instantly accessible to a viewer.

Group meetings are perfect vehicles for workshopping visuals. Every laboratory member, no matter how junior, can provide feedback on what they see and understand from a figure, as well as 
what changes would make the author's intended message clearer. For an excellent recent resource on developing and refining scientific visuals, I recommend Ref. [23].

\section{Lightning talks}

Lightning talks are informal, 60-second presentations given without a visual aid. They are often-but not always-given impromptu, similar to an "elevator pitch." The exercise combines well with discussions of related topics, like audience and message refinement, and provides speakers with a chance to practice developing skills in a low-risk environment while getting useful feedback from fellow participants [15]. The "Three Minute Thesis" competitions held at universities around the world represent a similar idea and may be attractive for some students as a venue for practicing their communication skills [24]. Within the fluids community, the "flash presentations" introduced at the 2019 APS DFD meeting are another such opportunity.

With this and other exercises, sticking with strict scientific topics is not necessary. A student might instead explain bicycle maintenance or why they prefer PYTHON over MATLAB.

\section{Revising for clarity and structure}

Sometimes revision involves digging into sentence- and paragraph-level constructions to improve clarity, brevity, or logical flow. Such exercises may be especially helpful for non-native speakers of English, but native speakers will benefit as well. Scientific writing guides like [9] contain excellent, premade revision exercises. For higher-level issues or more free-form exercises, Ref. [10] also provides many options easily implemented at the group or individual level.

\section{Critically evaluating science communication}

Groups often form journal clubs to review recent articles. Rather than focusing solely on the article's technical content, these groups can also examine how and how well the authors communicate. Do the authors' arguments flow clearly and logically? Can the figures stand separate from the text? What style and structure do the authors' use? Is the language overly complicated or clear and concise? Discussions like these help students identify and address weaknesses in their own work without the potential discomfort of presenting themselves [25]. The exercise can also highlight good examples of science communication that participants can emulate in their own work.

\section{Peer review}

Numerous studies show the value of peer review-students evaluating and providing feedback on one another's work-in improving communication skills and reducing the evaluation load on instructors $[9,14,15,24]$. Such active, collaborative learning mimics best practices in science communication training [15], helps students identify and refine their own methods [25], and can foster valuable mentorship roles between junior and senior students [11]. Even among undergraduate engineers, Nelson [25] found that students struggling with their own communication skills were often able to provide others with sound advice. Simpson et al. [11] describe successful department-level communication initiatives that prompted graduate students to form their own peer review networks.

When fostering peer review or implementing many of the exercises described above, it is important to emphasize constructive critique [26]. Combative attitudes and singling out individuals make the presenter feel attacked and defensive-not a useful environment for improvement. For this reason, I highly encourage collaborative attitudes toward revision and critique.

\section{CONCLUSION}

The interdisciplinary nature and visual complexity inherent to fluid dynamics makes communication skills critical for every student and practitioner. Mastering these skills requires frequent, deliberate practice, but integrating such practice into everyday research activities need not be 
difficult or onerous. Both research groups and informal groups can pursue simple, regular communication exercises and training that help all participants improve their skills.

Part of improving these skills is recognizing the process of science communication, which consists of four stages: (1) identifying big-picture issues around goals, audience, and message; (2) constructing a first version of the product; (3) evaluating and refining the product based on the bigger picture and outside feedback; and (4) reflecting on the lessons learned during the project. Of these stages, communicators often struggle most with the first and third. To this end, I have suggested multiple exercises aimed at planning, message refinement, revision, and critique. My hope is that these resources serve as a springboard for those looking to adopt or refine their scientific communication lifestyle.

\section{ACKNOWLEDGMENTS}

First and foremost, I thank J. Hertzberg, D. Hu, and J. Aurnou for their vocal support both of this paper and the talk that preceded it. Thanks are owed also to F. C. Frankel, with whom I first developed my concept of the science communication process, and A. Athanassiadis, who introduced me to the Half-Life Your Message exercise. I also thank G. Durey and D. Hu for granting permission to use their work as examples.

[1] A. T. Kirkpatrick, S. Danielson, R. O. Warrington, R. N. Smith, K. A. Thole, W. J. Wepfer, and T. Perry, Vision 2030: Creating the Future of Mechanical Engineering Education, ASEE Annual Conference and Exposition (ASEE, Vancouver, 2011).

[2] J. A. Donnel, B. M. Aller, M. P. Alley, and A. A. Kedrowicz, Why industry says that engineering graduates have poor communication skills: What the literature says, ASEE Annual Conference and Exposition (ASEE, Vancouver, 2011), pp. 22.1687.1-22.1687.13.

[3] P. Lappalainen, Communication as part of the engineering skills set, Eur. J. Eng. Educ. 34, 123, (2009).

[4] T. W. Burns, D. J. O'Conner, and S. M. Stocklmayar, Science communication: A contemporary definition, Public Underst. Sci. 12, 183, (2003).

[5] B. Trench and M. Bucchi, Science communication, an emerging discipline, J. Sci. Commun. 09, 1 (2010).

[6] R. T. Kellogg and A. P. Whiteford, Training advanced writing skills: the case for deliberate practice, Educ. Psychol. 44, 250 (2009).

[7] This model was initially developed in collaboration with Felice C. Frankel for a joint talk we gave at the Massachusetts Institute of Technology in 2016, and I have continued developing the concept independently since then.

[8] C. Cormick, The Science of Communicating Science (CSIRO, Clayton, Victoria, Australia, 2019).

[9] A. E. Greene, Writing Science in Plain English (The University of Chicago Press, Chicago, 2013).

[10] S. B. Heard, The Scientist's Guide to Writing (Princeton University Press, Princeton, 2016).

[11] S. Simpson, R. Clemens, D. R. Killingsworth, and J. D. Ford, Creating a culture of communication: A graduate-level STEM communication fellows program at a science and engineering university, Across the Disciplines 12, (2015).

[12] A. K. Dickerson, Z. G. Mills, and D. L. Hu, Wet mammals shake at tuned frequencies to dry, J. R. Soc. Interface 9, 3208 (2012).

[13] D. L. Hu, How to Walk on Water and Climb up Walls (Princeton University Press, Princeton, 2018).

[14] D. L. Hu, Confessions of a wasteful scientist, Sci. Am. Guest Blog (2016), https://blogs. scientificamerican.com/guest-blog/confessions-of-a-wasteful-scientist/.

[15] J. Silva and K. Bultitude, Best practice in communications training for public engagement with science, technology, engineering and mathematics, J. Sci. Commun. 08, 1 (2009).

[16] E. L. Aurbach, K. E. Prater, B. Patterson, and B. J. Zikmund-Fisher, Half-life your message: A quick, flexible tool for message discovery, Sci. Commun. 40, 669 (2018).

[17] N. Sharp, Half-Life your message timer, https://youtu.be/jD6EF5QIZi4. 
[18] N. Sharp, FYFD: tips for connecting with broader audiences, in APS DFD Annual Meeting (APS, Atlanta, GA, 2018).

[19] N. Sharp, Using the hero's journey to communicate science, https://youtu.be/9hSDnjyVC8o.

[20] The open notebook, https://www.theopennotebook.com.

[21] J. Hart, Storycraft (The University of Chicago Press, Chicago, 2011).

[22] G. Durey, H. Kwon, Q. Magdelaine, M. Casiulis, J. Mazet, L. Keiser, H. Bense, J. Bico, P. Colinet, and E. Reyssat, Marangoni bursting: evaporation-induced emulsification of a two-component droplet, 2017 Gallery of Fluid Motion, 2017, doi: 10.1103/APS.DFD.2017.GFM.V0020.

[23] F. C. Frankel, Picturing Science and Engineering (MIT Press, Cambridge, MA, 2018).

[24] Three minute thesis, http://threeminutethesis.org.

[25] S. Nelson, Teaching collaborative writing and peer review techniques to engineering and technology undergraduates, in Proceedings of the 30th ASEE/IEEE Frontiers in Education Conference (IEEE, Kansas, 2000), Vol. 2, pp. S2B/1-S2B/5.

[26] L. Lerman and J. Borstel, Critical Response Process: A Method for Getting Useful Feedback on Anything you Make, from Dance to Dessert (Dance Exchange, Inc, Takoma Park, 2003). 